\title{
Gene Expression Based Computation Methods for Alzheimer's Disease Progression using Hippocampal Volume Loss and MMSE Scores *
}

\author{
Aydin Saribudak \\ The City College of New York \\ Electrical Engineering Dept. \\ New York, NY, 10031 \\ asaribu000@citymail.cuny.edu
}

\author{
Adarsha A. Subick \\ The City College of New York \\ Electrical Engineering Dept. \\ New York, NY, 10031 \\ asubick00@ citymail.cuny.edu
}

\author{
Joshua A. Rutta \\ The City College of New York \\ Electrical Engineering Dept. \\ New York, NY, 10031 \\ jrutta000@ citymail.cuny.edu
}

\author{
M. Ümit Uyar \\ The City College of New York \\ Electrical Engineering Dept. \\ New York, NY, 10031 \\ uyar@ccny.cuny.edu
}

\author{
The Alzheimer's \\ Disease \\ Neuroimaging \\ Initiative ${ }^{\dagger}$
}

\begin{abstract}
Personalized relevance parameterization methods (PReP-AD) based on artificial intelligence computation techniques are introduced to investigate the impact of gene expressions on Alzheimer's disease (AD) progression. Our PReP-AD methods make use of the expressions of the genes that affect AD-related protein biomarkers (e.g., $A \beta_{1-42}$ and tau proteins), mini mental state examination (MMSE) scores and hippocampal volume measurements from ADNI database for the patients with mild cognitive impairment (MCI), an intermediate stage from normal cognition to AD. For MCI patients, disease progression is computed with PReP-AD-MMSE and PReP-AD-HVL methods, where the former utilizes the change in MMSE scores and the latter based on the rate of hippocampal volume loss over time. The performance of both methods are assessed with an algorithm implemented using leave-one-out-cross-validation (LOOCV). The cognitive changes of AD patients with MCI stage are detected with
\end{abstract}

\footnotetext{
* The initial research used in this work was supported by U.S. Army Communications-Electronics RD\&E Center contracts W15P7T-09CS021 and W15P7T-06-C-P217, and by the NSF grants ECCS0421159, CNS-0619577 and IIP-1265265. The contents of this document represent the views of the authors and are not necessarily the official views of, or are endorsed by, the U.S. Government, Department of Defense, Department of the Army or the U.S. Army Communications-Electronics RD\&E Center.

$\dagger$ Data used in preparation of this article were obtained from the Alzheimer's Disease Neuroimaging Initiative (ADNI) database (adni.loni.usc.edu). As such, the investigators within the ADNI contributed to the design and implementation of ADNI and/or provided data but did not participate in analysis or writing of this report. A complete listing of ADNI investigators can be found at: http: $\backslash \backslash$ adni.loni.usc.edu $\backslash$ wp-content $\backslash$ uploads $\backslash$ how_to_apply $\backslash$ ADNI_ Acknowledgement_List.pdf
}

Permission to make digital or hard copies of all or part of this work for personal or classroom use is granted without fee provided that copies are not made or distributed for profit or commercial advantage and that copies bear this notice and the full citation on the first page. Copyrights for components of this work owned by others than the author(s) must be honored. Abstracting with credit is permitted. To copy otherwise, or republish, to post on servers or to redistribute to lists, requires prior specific permission and/or a fee. Request permissions from permissions@acm.org. $B C B$ '16, October 2-5, 2016, Seattle, WA, USA

Copyright is held by the owner/author(s). Publication rights licensed to ACM. ACM 978-1-4503-4225-4/16/10 ...\$15.00.

DOI: http://dx.doi.org/10.1145/2975167.2991037. both our MMSE score and hippocampal volume based computation methods. We observe an average error rate of $4.8 \%$ with PRePAD-MMSE over a 72 -month period and $1.63 \%$ with PReP-AD-HVL over 12 months. The promising results indicate that artificial intelligence based computation methods can be utilized to build decision support tools for AD progression.

\section{Categories and Subject Descriptors}

G.4 [Mathematical Software]: Algorithm design and analysis ; J.3 [Life and Medical Sciences]: Biology and genetics, medical information systems

\section{General Terms}

Algorithms, Measurement, Design

\section{Keywords}

Alzheimer's disease, gene expressions, hippocampal volume loss, MMSE scores, mild cognitive impairment, MCI, AD biomarkers

\section{INTRODUCTION}

Alzheimer's disease (AD), the primary cause of age related dementia, is associated with a decline in cognitive abilities due to irreversible deterioration of brain functions [1]. Cognitive impairments of elderly people, such as loss of memory, language and judgmental deficits, are main symptoms of $\mathrm{AD}$ [2]. The intermediate stage that AD patients experience while passing through a gradual transition from normal cognition to $\mathrm{AD}$ is termed Mild cognitive Impairment (MCI) [3]. An increasing need for computational methods emerges to evaluate cognitive impairment and diagnose dementia in preclinical or early clinical stages of AD, including MCI stage [4].

The cognitive decline in aging and AD can be linked to hippocampal volume atrophy with the assessment of repeated 3D MRI scans [5]. For example, Jack et al. [3] reported a significant difference in annualized percentage change of hippocampal volume among AD patient groups based on their MRI examinations. Another measure of cognitive impairment in AD type dementia is the Mini-Mental State Examination (MMSE), which is a 30-point questionnaire [1]. With MMSE, the cognitive and behavioral status of patients, such as attention, concentration and short term recall, are 
assessed, where a lower score implies greater impairment [6]. Typically MMSE scores of AD patients decrease with the progression of the disease [7].

Laske et al. have noted the relevance of recent neuropathological, biochemical and neuroimaging findings with early diagnosis of $\mathrm{AD}$ indicating the importance of biomarkers for $\mathrm{AD}$ progression [8]. The 42 aminoacid form of $\beta$-amyloid $\left(A \beta_{1-42}\right)$ and tau protein$\mathrm{s}$, which are indicative of tangle formations in cerebrospinal fluid (CSF), are identified as AD protein biomarkers in numerous studies reported in [9]. In [10], quantitative and qualitative alterations of gene expressions are analyzed to identify the biomarkers reflecting underlying biological pathways for $\mathrm{AD}$.

In this paper, we utilize expressions of genes that are reported to be related to $\mathrm{AD}$ based on analysis of gene expression profiles and blood samples $[8,10,11,12]$. In addition, hippocampal volume measurements and MMSE scores for the patients with MCI status from Alzheimer's Disease Neuroimaging Initiative (ADNI) database [13] are included to compute disease progression. In ADNI, volume measurements are collected at three distinct time points a year, whereas MMSE scores are obtained for a 72-month period. Our personalized relevance parametrization (PReP-AD) methods are built for AD progression using artificial intelligence based computation techniques from our earlier reserach $[14,15,16]$. Here, we introduce two methods, namely PReP-AD-MMSE and PReP-ADHVL, to compute disease progression with MMSE scores and hippocampal volume mesaurements, respectively. To identify the unbiased impact of gene expressions on the time course of disease, MCI patients who were not exposed to any AD drug therapy (e.g., donepezil and memantine) are selected as subjects for both methods

To evaluate the performance of PReP-AD methods, we implement a validation algorithm using leave-one-out-cross-validation (LOOCV) technique. A relatively small average error rate obtained for both PReP-AD methods indicate that computational methods based on gene expressions and hippocampal measurements may be useful to build decision support tools for AD progression.

The rest of this article is organized as follows. In Sec. 2, a brief introduction is provided for related work reported in literature. MMSE and hippocampal volume measurements for AD are explained in Sec. 3. Sec. 4 describes PReP-AD algorithms. Analytical results and their interpretation are presented in Sec. 5.

\section{RELATED WORK}

Alzheimer's disease is a debilitating neurodegenerative disease characterized by the development of amyloid beta plaques and neurofibrillary tangles in the brain, as well as a decrease in hippocampal volume and onset of cognitive impairment and dementia [17]. Extensive work has been reported for AD in the fields of genetic, biomarker, imaging, and trajectory research. Blennow et al. [9] reported that an elevated level of total-tau (t-tau) in CSF is an indicator of neuronal degeneration and that phosphorylated-tau (p-tau) may relate to the development of neurofibrillary tangles. In [11], MRI images demonstrate the acceleration of hippocampal volume atrophy with the presence of apolipoprotein E (ApoE) allele $\varepsilon 4$ gene and decreased $\beta$-amyloid $\left(A \beta_{1-42}\right)$ concentrations. Laske et al. [8] identify 24 blood-based biomarkers as an alternative to measuring levels of $A \beta_{1-42}$, t-tau and $\mathrm{p}$-tau, which requires an invasive and painful extraction of CSF via a lumbar puncture.

Attempts have been made to develop a robust clinical rating scale to track AD progression. A comprehensive review, reported in [1], identified 68 scales that measure domains such as cognition, behavior, and quality of life, however none were found to adequately gauge multiple domains simultaneously and sensitive enough at different stages of the disease. One of the most common rating systems is MMSE [18, 19, 20], a 30-point test which requires approximately 5-10 minutes to administer for a patient. Based on the patient's correct answers to the test, an MMSE score is assigned to gauge short term recall, orientation to time and space, ability to follow commands, and other cognitive functions.

In [21], the effect of ApoE allele $\varepsilon 4$ gene on cognitive and functional abilities is examined using a linear declining pattern based on several metrics including MMSE scores. In [20], AD progression is predicted by quadratic and logarithmic curve fitting functions based on the relationship between declining rate and average value of MMSE scores over time. Other researchers describe AD progression by a trilinear model [22] and nonlinear functions [7], where the most rapid decline in MMSE scores occurs in midrange of the disease and less rapid decline during early and late stages. Stern et al. [7] estimate that the total period of decline in MMSE scores is about 15 years, where a rapid decline occurs after year 6 .

In our ongoing research, we study the effects of biomarkers on progression for cancer and neurodegenerative diseases. In [23], we compute tumor growth for breast cancer patients using their gene expressions and MRI images. In [24, 25], we examine the delay effect of anti-cancer agents on tumor growth and the amount of shrinkage in tumor mass with the administration of chemotherapy for a number of breast cancer cell lines implanted in xenograft mice models. We also applied spatial pattern analysis methods for kidney cancer xenograft pathology slides to compute patient-specific features based on artificial intelligence techniques [16, 26]. Computing morphological features of in-vitro cell based assays which are incubated with human colon carcinoma cell lines, we examine spatial heterogeneity as a biomarker for drug efficacy measurements in $[27,28]$. In this paper, we aim to compute disease progression for MCI transition stage of $\mathrm{AD}$, which is from normal cognition to severe disease, based on gene expressions. Our computation methods are built on MMSE scores and hippocampal volume atrophy collected from AD patients in MCI stage.

\section{ALZHEIMER'S DISEASE}

Alzheimer's disease, contributing to $60 \%$ of dementia cases, affects approximately 35 million people worldwide with the total number of $\mathrm{AD}$ cases projected to more than triple by $2050[4,29]$. It is also estimated that more than 4 million people suffer from dementia in the United States alone [30]. Due to the fact that brain changes begin 20 or more years before AD symptoms appear, characterization of AD progression gains more importance [31].

$\mathrm{ADNI}$ is an longitudinal study for diagnosis of $\mathrm{AD}$ and analysis of treatment effects in AD type dementia [30]. In ADNI database, neuroimaging, CSF and blood-based biomarkers are collected from a large number of patients representing all stages of AD. This information is made available to aid in the discovery of biomarkers that can be used to most effectively diagnose and monitor the effects of treatment for AD. To evaluate cognitive decline, clinical measurements such as hippocampal volume measurements and neuropsychological tests such as MMSE were periodically administered to patients as part of the ADNI study [30]. Gene expressions, MMSE scores and hippocampal volume measurements used in this paper are obtained from ADNI database for the AD patients with MCI stage.

\subsection{MMSE Scores}

MMSE is a clinical tool designed as a questionnaire to assess cognitive state of patients [1]. MMSE is not used to identify the particular forms of dementia that a patient may have, but, instead, it determines the level of cognitive impairment and documents cognitive change in AD type dementia over time [1, 18, 32]. Consisting of 11 
questions in 7 different categories, the MMSE has a maximum score of 30 points to classify patients into three disease levels, namely, normal cognition, mild and severe cognitive impairment $[18,32]$. To focus on the cognitive aspects of mental functions for AD patients, abnormal mental experience and mood concerned questions are not included in the MMSE questionnaire [18]. In this paper, we use the change in MMSE scores for MCI patients over time as a metric for $\mathrm{AD}$ progression.

\subsection{Hippocampus Volume Measurements}

Magnetic resonance imaging (MRI) scans from AD patients are analyzed in longitudinal research studies to track the changes in brain structure over time [17]. The rate of hippocampal volume loss calculated from sequential MRI scans of the same individual is higher in AD patients than in control groups [33, 34]. Hippocampal atrophy could be assessed with MRI scans to decide whether the disease level declines or remains stable over a period of time [3].

Brain and specifically hippocampal volume measurements of $\mathrm{AD}$ patients are reported in ADNI database for early and late MCI, and control patients. For each individual, a baseline scan and two additional follow-up scans are included as high resolution MRI images using 3-Tesla MRI scanners. In Fig. 1, we present a sample MRI image of a 85-year old male MCI patient's brain, whose subject ID is reported as $005-\mathrm{S}-0448$ in the database. The brain images shown in Fig. 1 are captured from the axial (left pane), the coronal (middle pane) and the sagittal (right pane) plane corresponding to the an example point of interest, which is marked with horizontal and vertical axis lines on each image. To provide a better view, the interest point is selected in the hippocampus region, which is highlighted in blue with the skull digitally removed. The left- and right-most positions of the skull are 54 and 195 voxels for axial view, 55 and 214 voxels for coronal view and 21 and 137 voxels for sagittal view. In this paper, in addition to the change in MMSE scores for MCI patients, we also use the rate of change in hippocampal volume in MRI scans over time as a metric for AD progression.

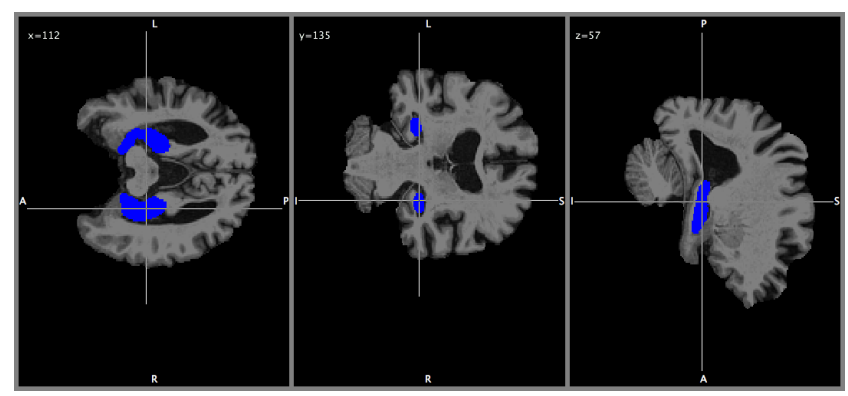

Figure 1: MRI brain scan of patient 005-S-0448, an 85-year old male MCI patient, captured at baseline visit. The skull has been digitally removed and hippocampus is highlighted in blue. The rate of change in hippocampal volume between sequential visits is used as a metric for disease progression. The images shown are from the axial (left pane), the coronal (middle pane) and the sagittal (right pane) plane of the point of interest with the position of $x=112$ voxels, $y=135$ voxels, and $\mathrm{z}=57$ voxels.

\section{PREP-AD FOR DISEASE PROGRESSION}

In our research, we have examined the relationship between gene expressions and tumor growth and shrinkage for cancer patients. Using gene expressions measured from a set of breast cancer patients reported in I-SPY 1 TRIAL database [35] and their MRI images in NBIA database [36], we have computed personalized tu- mor growth and shrinkage parameters. In [23], we analyze tumor growth when there is no drug administration by computing exponential-linear model parameters. The delay and shrinkage effect of anti-cancer agents on tumor proliferation is examined by computing transit compartmental PK-PD model parameters in [24, 25]. Here, we introduce our personalized parametrization methods, namely PREP-AD-MMSE and PREP-AD-HVL, based on MMSE scores and hippocampus volume loss, respectively.

\subsection{Alzheimer's Disease Related Genes}

Our PReP-AD methods make use of the expressions of the genes that affect $\mathrm{AD}$-related protein biomarkers (e.g., $A \beta_{1-42}$ and tau proteins) reported in literature $[8,10,11,12]$. These genes are listed in Table 1 with their Human Genome Organisation (HUGO) symbol names. The expression values of these AD-related genes are obtained from ADNI database [13] for the patients with MCI status, excluding the patients who were administered any AD drugs. We use the mean expression value for the genes belonging to the same family. For example, the mean expression values for ADIPOR 1 and ADIPOR2 genes are used for ADIPOR family. Note that, in this paper, gene families are also referred to as genes for simplification.

Table 1: HUGO SYMBOLS OF ALZHEIMER's DISEASE GENES

\begin{tabular}{|c|c|c|c|c|}
\hline \multicolumn{5}{|c|}{33 genes from [10] } \\
\hline ADD3 & AGT & APLP1 & C4BPA & C4BPB \\
\hline CD81 & CHN2 & CLU & CLUH & CLUL1 \\
\hline DMPK & DTNA & EEF1A1 & GSTM2 & GSTM2P1 \\
\hline HBB & HBG2 & IGHMBP2 & INHBB & KRT8 \\
\hline LIMS2 & LRRC4B & MT1G & OSBPL3 & PCSK1N \\
\hline PLEKHB1 & PLP1 & PRDX1 & PTS & RANGAP1 \\
\hline RAP1GDS1 & RPL31 & TSPAN3 & & \\
\hline \multicolumn{5}{|c|}{58 genes from [8] } \\
\hline ACSF2 & ACSF3 & ADIPOQ & ADIPOR1 & ADIPOR2 \\
\hline APP & BDNF & CCL5 & CCL22 & CSF1 \\
\hline CSF1R & CSF2 & CSF2RA & CSF2RB & CSF3 \\
\hline CSF3R & EGFL6 & EGFL7 & EGFL8 & EGFLAM \\
\hline EGFR & HBEGF & ICAM1 & LEP & LEPR \\
\hline LEPRE1 & LEPREL1 & LEPREL2 & LEPREL4 & LEPROT \\
\hline LEPROTL1 & MEGF6 & MEGF8 & MEGF9 & MEGF10 \\
\hline MEGF11 & OLR1 & PDGFA & PDGFB & PDGFC \\
\hline PDGFD & PDGFRA & PDGFRB & PDGFRL & SELPLG \\
\hline TF & TNFAIP1 & TNFAIP2 & TNFAIP3 & TNFAIP6 \\
\hline TNFAIP8 & TNFAIP8L1 & TNFAIP8L2 & TNFAIP8L3 & VEGFA \\
\hline VEGFB & VEGFC & VWF & & \\
\hline \multicolumn{5}{|c|}{$\begin{array}{l}\text { ApoE (tau-protein activator) and } \\
\text { MAPT (microtubule-associated protein tau) from [11] }\end{array}$} \\
\hline APOE & MAPT & & & \\
\hline \multicolumn{5}{|c|}{3 genes from [12] } \\
\hline SOD1 & SOD2 & SOD3 & & \\
\hline
\end{tabular}

\subsection{Genetic Data Vector}

Genetic data vector $\Theta_{\beta_{u}}$ is defined for a patient $\beta_{u}$ using gene expressions (collected only once at the beginning of the study), their correlations and an offset constant. $G$ matrix with dimensions of $n x r$ is formed with gene expressions from $n$ MCI patients and $r$ AD-related genes. To represent a relationship among a set of gene expressions, we identify a correlated gene set $\mathbb{C}$ stated as

$$
\mathfrak{C}_{k}=\left\{g_{i} \in \Gamma: \quad C\left(G_{i}, G_{j}\right) \geq \tau, \forall g_{j} \in \mathfrak{C}_{k} \backslash g_{i}\right\}
$$

where $\Gamma$ is the set for AD-related genes and $\tau$ is the threshold parameter. $C\left(G_{i}, G_{j}\right)$ is the correlation between column vectors $G_{i}$ and $G_{j}$ of matrix $G$. Correlated gene set $\mathfrak{C}_{k}$ is a unique set, where 
a corresponding parameter $\theta_{k}$ is computed as

$$
\theta_{k}=\prod_{g_{i} \in \mathfrak{C}_{k}} g_{i}
$$

In this study, to construct set $\mathfrak{C}_{k}$ for MCI patients, correlated gene sets are built for pairs of genes (i.e., $\left|\mathfrak{C}_{k}\right|=2$ ). The threshold constant $\tau$ is determined to limit the number of correlation parameters within a predetermined range. For PReP-AD-MMSE method, $\tau=0.72$ for 115 patients and $\tau=0.6$ for 175 patients in PReP-ADHVL method.

Using the expressions of AD-related genes and the correlation parameters for a patient $\beta_{u}$, genetic data vector $\Theta_{\beta_{u}}$ is stated as

$$
\Theta_{\beta_{u}}=<c, g_{1}, \cdots, g_{r}, \theta_{1}, \ldots, \theta_{s}>
$$

where $c$ is the offset constant, $g_{i}$ is the expression of $i^{\text {th }}$ gene, $\theta_{j}$ is the $j^{\text {th }}$ correlation parameter, and $r$ and $s$ are the number of genes and correlation parameters, respectively. Note that $\Theta_{\beta_{u}}$ is a column vector of genetic data matrix $\Theta$ which is constructed for all patients.

Various studies report a linear decline in patient cognitive skills as time progresses during MCI stage [7, 22]. In PReP-AD methods, linear decline parameters are calculated for the MCI patients whose MMSE scores and hippocampus volume measurements are available in ADNI database. For a patient $\beta_{u}$, vector $P_{\beta_{u}}$ is stated as $P_{\beta_{u}}=\left\langle\mathfrak{p}_{1, u}, \mathfrak{p}_{2, u}, \cdots, \mathfrak{p}_{\mu, u}\right\rangle$, where $\mathfrak{p}_{v, u}$ is the $v^{\text {th }}$ parameter and integer $v \in[1 \mu]$. To compute disease progression for patient $\beta_{u}$, our model equation can be formulated as

$$
P_{\beta_{u}}=\mathfrak{A}_{A D} \cdot \Theta_{\beta_{u}}
$$

where $\mathfrak{A}_{A D}=\left[a_{i j}\right]$ is the $\mu \times m$ dimensional genetic accordance matrix constructed for $\mu$ number of progression parameters and $m$ number of elements of genetic data vector $\Theta_{\beta_{u}}$. Here, the relationship between parameters can be expressed as $m=r+s+1$.

\subsection{Genetic Accordance Matrix for AD}

To analyze the effect of gene expressions on the behavior of disease progression, we define array $P^{v}$ consisting of progression parameters from $n$ patients as

$$
\widetilde{P}^{v}=\left(\tilde{\mathfrak{p}}_{v, 1}, \tilde{\mathfrak{p}}_{v, 2}, \cdots, \tilde{\mathfrak{p}}_{v, n}\right)
$$

where $\tilde{\mathfrak{p}}_{v, u}$ is the estimated value of the $v^{\text {th }}$ progression parameter for patient $\beta_{u}$. Using genetic information and the parameters, each row vector of coefficient matrix $\mathfrak{A}_{A D}$ is computed as

$$
\mathfrak{A}_{A D}^{v}=\widetilde{P}^{v} \cdot\left[\begin{array}{c}
C \\
\Theta_{D}
\end{array}\right]^{-1}
$$

where $C$ is a row vector with offset constant $c$ for $n$ patients and $\Theta_{D}$ is the reduced from of genetic data matrix $\Theta$.

To determine the impact of individual gene differences, we utilize differential evolution (DE) based artificial intelligence techniques from our earlier research which are known to perform better than traditional methods due to flexibility of fitness functions and inclusion of non-linear relationships [14, 15, 16, 37]. Genetic accordance matrix $\mathfrak{A}_{A D}$ is constructed for both PReP-AD methods as illustrated with Algorithm 1. Progression parameters from MMSE scores and hippocampus volume measurements (i.e., the rates of change in disease level) are inputs for PReP-AD-MMSE and PRePAD-HVL methods, respectively. The population size, number of generations and DE-based constants are also inputs for Algorith$\mathrm{m} 1$. First, we construct genetic data vector $\Theta_{\beta_{u}}$ for all patients as shown at line 1 . For each progression parameter $\mathfrak{p}_{v}$ of $\mathrm{AD}$, we then instantiate an initial population of candidate vectors $\vec{a}^{1}$ as shown at line 3 of Algorithm 1. Here, since DE algorithm does not require an initial knowledge to track the fittest solution in the solution space [38], initial population of candidate solutions is declared randomly. At line 4, the fitness array of initial population is calculated. The fittest candidate vector array $\vec{a}_{v, \text { best }}$ and the best fitness array $F\left(\vec{a}_{v, \text { best }}\right)$ are initialized at line 5 .

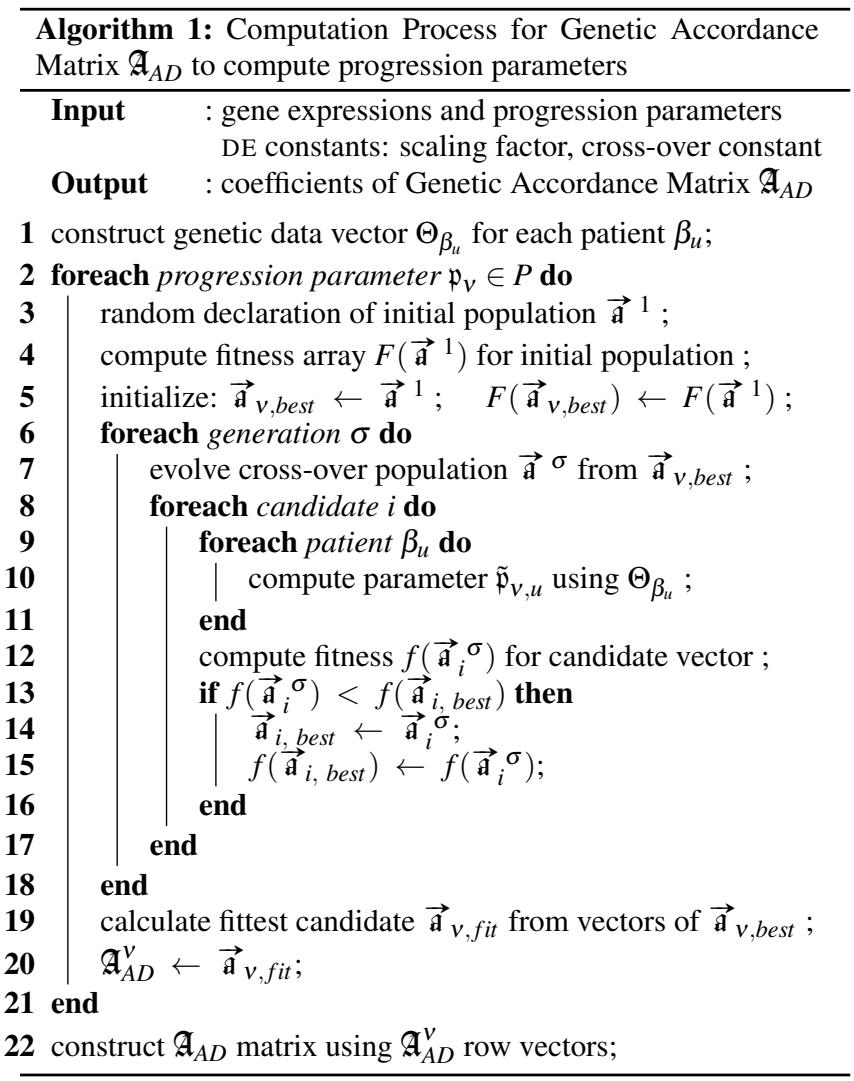

For each generation, cross-over population is calculated from the best candidate vector array (line 7) based on DE algorithm constants. Here, scaling factor and cross over constant are utilized to search the solution space based on exploitation and exploration features of DE-based computation. The exploitation feature of DE performs a thorough search over a local region, while exploration feature searches randomly over the global solution space [38].

Using the coefficients of candidate vector, progression parameters are computed (lines 9-11). Algorithm 1 computes the fitness of the candidate solution of $f\left(\vec{a}_{i}{ }^{\sigma}\right)$ at line 12 formulated as

$$
f\left(\overrightarrow{\boldsymbol{a}}_{i}{ }^{\sigma}\right)=\frac{1}{n} \cdot \sqrt{\sum_{u=1}^{n}\left(\frac{\mathfrak{p}_{v, u}-\tilde{\mathfrak{p}}_{v, u}}{\mathfrak{p}_{v, u}}\right)^{2}}
$$

where $\mathfrak{p}_{v, u}$ and $\tilde{\mathfrak{p}}_{v, u}$ are the measured and estimated progression parameters, respectively, for patient $\beta_{u}$. To decrease the variance among error rates computed for MCI patients, the least square calculation of fitness function is weighted inversely with the magnitude of the measured progression parameter. At lines 13-16, the fittest vector $\overrightarrow{\mathfrak{a}}_{v \text {,best }}$ and the best fitness vector $F\left(\overrightarrow{\boldsymbol{a}}_{v, \text { best }}\right)$ are calculated for cross-over population. To converge to the fittest solution, the best population array $\vec{a}_{v \text {, best }}$ is evolved generation by generation. The fittest individual vector $\vec{a}_{v, \text { fit }}$ from the best population $\vec{a}_{v, \text { best }}$ 
shown at line 19 of Algorithm 1 can be found as:

$$
\overrightarrow{\mathfrak{a}}_{v, f i t}=\underset{\overrightarrow{\mathfrak{a}}_{i} \sigma \in \overrightarrow{\boldsymbol{a}}_{v, \text { best }}}{\operatorname{argmin}} f\left(\overrightarrow{\boldsymbol{a}}_{i}^{\sigma}\right)
$$

where $\overrightarrow{\mathfrak{a}}_{v, f i t}$ is declared as the row vector $\mathfrak{A}_{A D}^{v}$ (line 20). $\mathfrak{A}_{A D}$ matrix is constructed at line 22 using the row vectors computed for parameter $\mathfrak{p}_{v}$. With genetic accordance matrix $\mathfrak{A}_{A D}$ in Eq. (4), progression parameter $\mathfrak{p}_{v, u}$ for patient $\beta_{u}$ can be calculated as

$$
\mathfrak{p}_{v, u}=c \cdot a_{v, 0}+\sum_{i=1}^{r} a_{v, i} \cdot g_{i}+\sum_{j=1}^{s} a_{v,(j+r)} \cdot \theta_{j}
$$

where $a_{v, i}$ is the $i^{t h}$ coefficient of $v^{t h}$ row of matrix $\mathfrak{A}_{A D}^{v}$. Parameters c, $g_{i}$ and $\theta_{j}$ are all elements of genetic data vector $\Theta_{\beta_{u}}$ constructed for patient $\beta_{u}$ as defined in Eq. (3).

\subsection{Paired Difference Method}

There are 115 MCI patients in ADNI database that can be used in PReP-AD-MMSE method. On the other hand, for PReP-AD-HVL method, hippocampus volume measurements are available only for 26 MCI patients, where a decrease in measurements has been observed for 15 of them. To include the patients without reported volume measurements into our method, we build an algorithm that assigns progression parameters defined in Eq. (4) based on similarities of gene expressions between patients.

Algorithm 2 computes hippocampus volume based progression parameters for PReP-AD-HVL method using gene expressions and volume measurements as input parameters. The similarities of gene expressions between patient pairs are identified based on manhattan distance metric [39]. To determine the impact of individual gene differences, we utilize DE-based techniques [16].

In Algorithm 2, at line 1, we classify MCI patients into two groups, namely $V$ and $\bar{V}$, consisting of the patients with and without hippocampus volume measurements, respectively. Progression parameters for patients from set $V$ are calculated based on a linear decreasing behavior of hippocampus volume (line 3 ). The gene expression and progression parameter differences are calculated at lines 4-9 for each patient pair to build gene expression difference matrix $G_{\Delta}$ and parameter difference vector $P_{\Delta}$. At line 6 , calculation of row vector $G_{\Delta}^{i j}$ of matrix $G_{\Delta}$ is presented for patients $\beta_{i}$ and $\beta_{j}$, where $\phi^{\text {th }}$ element is the magnitude of gene $g_{\phi}$ expression difference (i.e., $\left.\left|g_{\phi, i}-g_{\phi, j}\right|\right)$. Similarly, vector $P_{\Delta}^{i j}$ constructed with the manhattan distance of progression parameters, where $\left|\mathfrak{p}_{v, i}-\mathfrak{p}_{v, j}\right|$ is the $v^{\text {th }}$ element of the vector for patients $\beta_{i}$ and $\beta_{j}$ (line 7). The relationship between gene expression and progression parameter differences can be formulated as

$$
P_{\Delta}=\left[E \mid G_{\Delta}\right] \cdot \vec{w}_{f i t}
$$

where $\vec{w}_{f i t}$ is the fittest weight, $E$ is a column vector with negative offset constants and $G_{\Delta}$ is a $\left(\begin{array}{l}n \\ 2\end{array}\right) \times r$ dimensional matrix for $n$ number of patients and $r$ number of genes.

In our paired difference computation method, $\vec{w}_{f i t}$ is computed by a DE-based technique. An initial population of weight vectors is defined randomly at line 10 and corresponding fitness array is computed at line 11 . The population is evolved generation by generation at lines 12 to 18 to calculate the fittest weight vector population $\vec{w}_{\text {best }}$. At line 19 , the fittest individual weight vector $\vec{w}_{\text {fit }}$ is calculated from $\vec{w}_{\text {best }}$ population.

Using the fittest weight vector, computed based on genetic similarity among patients, the progression parameters are calculated for set $\bar{V}$ at lines 20-29. First, we select a patient $\beta_{\bar{u}}$ randomly from set
$\bar{V}$ at line 21 . The computation of paired difference between $\beta_{\bar{u}}$ and a patient $\beta_{i} \in V$, as given in lines 22-24, can be formulated as

$$
f_{\delta}\left(\beta_{\bar{u}}, \beta_{i}\right)=w_{0} \cdot c+\sum_{g_{\phi} \in \Gamma} w_{i} \cdot\left|g_{\phi, \bar{u}}-g_{\phi, i}\right|
$$

where $\Gamma$ is the set of AD-related genes. At line 25, the most genetically similar patient $\beta_{u} \in V$ is calculated for patient $\beta_{\bar{u}}$ as

$$
\beta_{u}=\underset{\beta_{i} \in V}{\operatorname{argmin}} f_{\delta}\left(\beta_{\bar{u}}, \beta_{i}\right)
$$

At line 26, using the progression parameter $\mathfrak{p}_{v, u}$, parameter $\mathfrak{p}_{v, \bar{u}}$ is calculated as

$$
\mathfrak{p}_{v, \bar{u}}=\mathfrak{p}_{v, u} \cdot \begin{cases}{\left[1+\eta \cdot f_{\delta}\left(\beta_{\bar{u}}, \beta_{u}\right)\right]} & \text { if } \gamma \leq 0.5 \\ {\left[1-\eta \cdot f_{\delta}\left(\beta_{\bar{u}}, \beta_{u}\right)\right]} & \text { otherwise }\end{cases}
$$

where $\gamma \in\left[\begin{array}{ll}0 & 1\end{array}\right]$ is a random number and $\eta$ is the normalization constant defined to keep the value of parameter $\mathfrak{p}_{v, \bar{u}}$ at most $25 \%$ distinct to the parameter $\mathfrak{p}_{v, u}$ of the genetically similar patient. At line 27 patient $\beta_{\bar{u}}$ is removed from set $\bar{V}$ and, at line 28 , inserted into set $V$. This process is repeated until the parameters are calculated for all patients and no patient is left in set $\bar{V}$.

Algorithm 2: Computation of progression parameters for the patients without hippocampus volume measurement

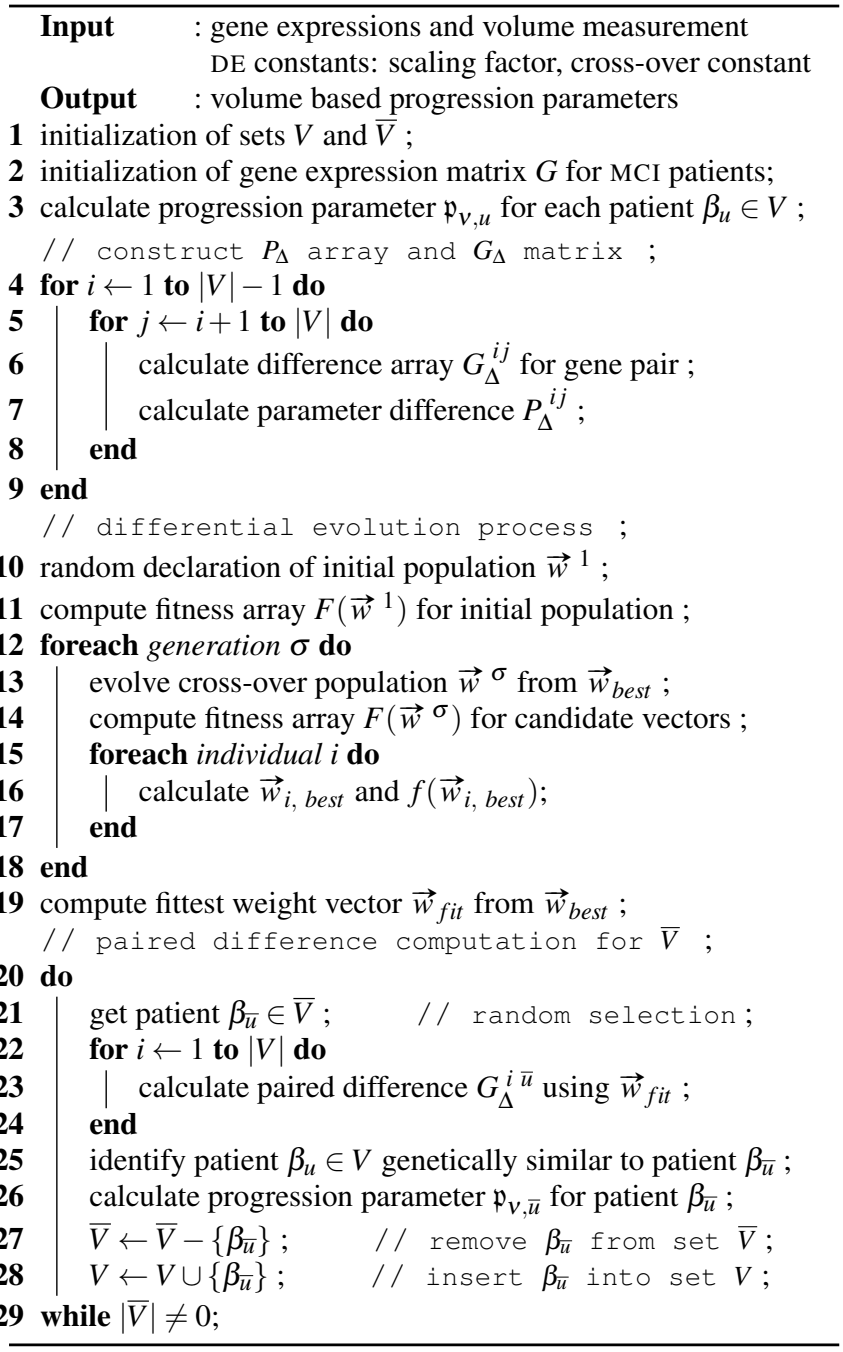




\subsection{Cross Validation for PReP-AD}

PReP-AD methods compute disease progression parameters using information from ADNI database for MCI patients by Algorithms 1 and 2. To compute error rates for disease progression with respect to MMSE scores and hippocampal volume measurements we built a validation algorithm (presented in Algorithm 3) based on leave-one-out-cross-validation (LOOCV) method [40].

In Algorithm 3, we first initialize matrix $\Theta$ with genetic data vectors and vector $P$ with progression parameters of $n$ MCI patients. For a patient $\beta_{u}$ (called a test patient), the genetic data vector $\Theta_{\beta_{u}}$ is built at line 4 and vector of progression parameters $P_{\beta_{u}}$ is calculated at line 5. At lines 6 and 7, we use gene expression and disease progression information of the remaining $(n-1)$ patients (called the training set) to build $\Theta_{R}$ and $P_{R}$ matrices, respectively. At line 8, training matrices $\Theta_{R}$ and $P_{R}$ are used in Algorithm 1 to compute the genetic accordance matrix $\mathfrak{A}_{A D}$ for the training set. For test patient $\beta_{u}$, using matrix $\mathfrak{A}_{A D}$ in Eq. (4), we compute vector $P_{\beta_{u}}^{e s t}$ of estimated progression parameters (line 9). Disease progression is then calculated at line 10 based on MMSE scores for PReP-ADMMSE or volume measurements for PReP-AD-HVL.

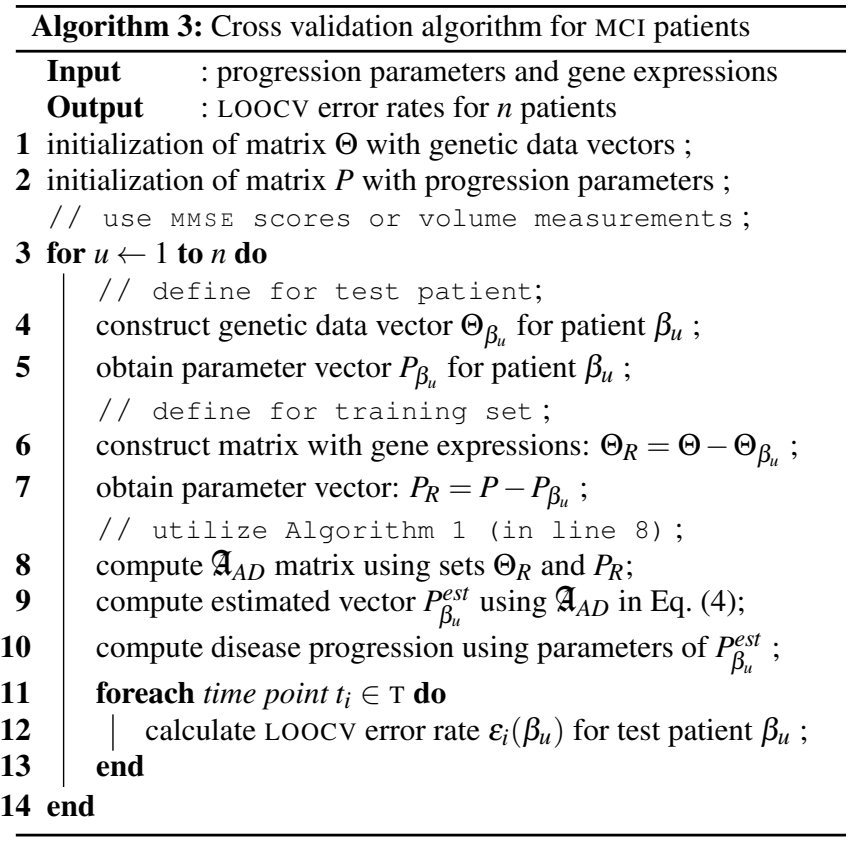

At lines 11 to 13 of Algorithm 3, LOOCV error rate $\varepsilon_{i}\left(\beta_{u}\right)$ is calculated for each time point $t_{i}$ using the disease progression parameters computed above for test patient $\beta_{u}$ as follows:

$$
\varepsilon_{i}\left(\beta_{u}\right)=\left|1-\frac{\widetilde{\mathrm{AD}_{u}}\left(t_{i}\right)}{\mathrm{AD}_{u}\left(t_{i}\right)}\right|
$$

where $\mathrm{AD}_{u}\left(t_{i}\right)$ and $\widetilde{\mathrm{AD}}_{u}\left(t_{i}\right)$ are the measured and computed MMSE scores, respectively, in PReP-AD-MMSE at time point $t_{i}$; similarly, $\mathrm{AD}_{u}\left(t_{i}\right)$ and $\widetilde{\mathrm{AD}}_{u}\left(t_{i}\right)$ correspond to the measured and computed hippocampal volumes in PReP-AD-HVL.

Although LOOCV avoids the problem of over-fitting of results to the existing patient data, the results of Algorithm 3 can be further improved by increasing the number of patients.

\section{ANALYTICAL RESULTS}

In this study, we build gene expression based personalized parameterization methods to compute disease progression for $\mathrm{MCI}$ patients. In addition to gene expressions, MMSE scores from 115 patients and hippocampal volume data from 26 patients are utilized. Using genetic similarities between patients, Algorithm 2 generated volume based progression parameters, which resulted in a decreasing disease progression for 175 patients. The patients who received AD drugs (e.g., donepezil and galantamine) are disregarded to study unbiased impact of gene expressions on disease progression.

As described earlier, using measurements in ADNI database for MCI patients and the volume measurements calculated by Algorithm 2, PReP-AD methods compute linear decreasing rates for MMSE scores and hippocampal volumes. Then, with Algorithm 3, LOOCV error rates are computed. where we observe an average error rate of $4.8 \%$ with PReP-AD-MMSE over a 72-month period and $1.63 \%$ with PReP-AD-HVL over 12 months of disease progression.

\subsection{PReP-AD-MMSE Results}

Using the gene expressions and the MMSE scores for $115 \mathrm{MCI}$ patients, we compute a decreasing progression for 54 patients, a stable progression (i.e., a change of \pm 1 point in 72 months) for 52 patients and an increasing behavior for 9 patients (which incorrectly implies that those 9 patients recover from $\mathrm{AD}$ ). Including those 9 patients, we obtain an average error rate of $4.8 \%$ for 72 months.

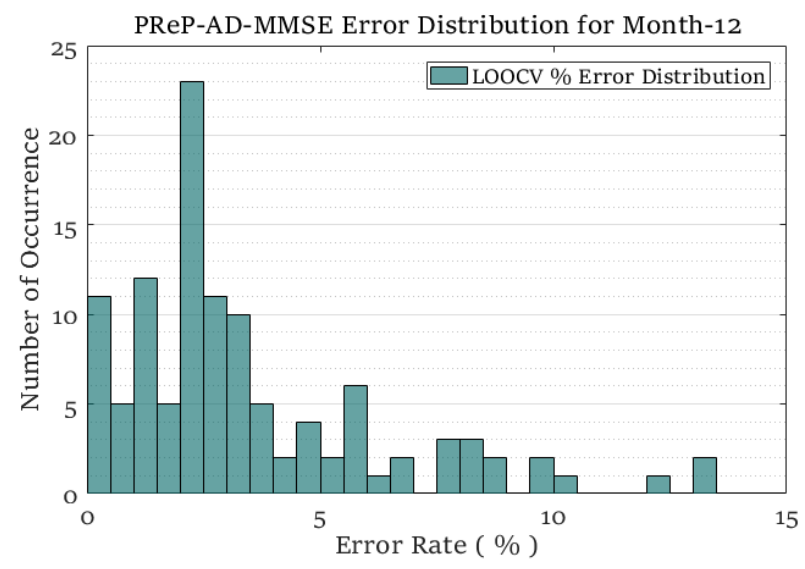

(a)

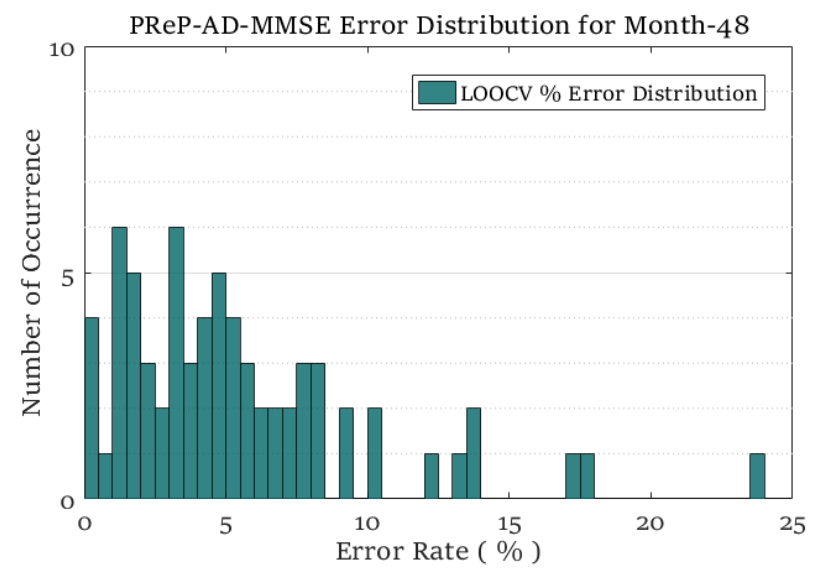

(b)

Figure 2: Histogram of LOOCV percentage error rates of PReP-ADMMSE method for $115 \mathrm{MCI}$ patients at month 12 in (a) and for 69 MCI patients at month 48 in (b) 
Histograms of LOOCV percentage error rates for PReP-AD-MMSE method are presented in Figs. $2 \mathrm{a}$ and $2 \mathrm{~b}$ for months 12 and 48, respectively. In Fig. 2a, an average error rate of $3.47 \%$ with a standard deviation of $2.82 \%$ is obtained for 115 MCI patients whose computed disease progression is stable or declining. The distribution of error rates for month 48 is shown in Fig. $2 b$ for 69 patients, where the average error is $5.49 \%$ with a standard deviation of $4.55 \%$.

Fig. 3 illustrates the distribution of error rates of PReP-AD-MMSE for multiple time points with a boxplot graph. In Fig. 3, the box generated for each time point represents the range of error rates from the first quartile to the third. The maximum and minimum values are marked with the horizontal black lines, and the median with a horizontal red line. The outliers, which are the points beyond 1.5 times of interquartile range (IQR) from the first and the third quartiles, are shown as plus signs in red.

In Table 2, the percentage LOOCV error rate statistics, including the median, IQR and the number of outliers, are listed corresponding to the boxplot graph in Fig. 3. From boxplot graph of PReP-AD-MMSE and from error rate statistics given in Table 2, we observe that the error rate increases as time progresses, where the amount of increase from month 36 to 48 is the largest.

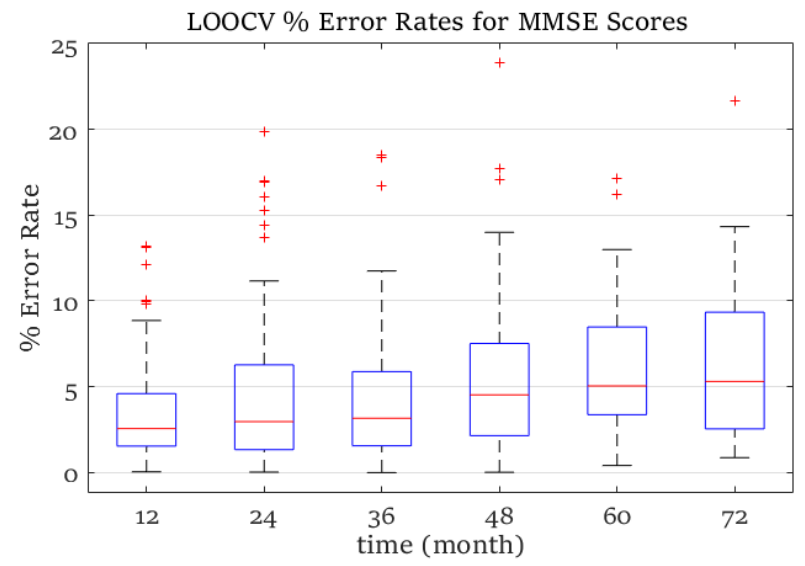

Figure 3: Boxplot graph of percentage LOOCV error rates of PRePAD-MMSE for a 72-month period

Table 2: Number of patients, mean error rate and percentage LOOCV error rate statistics from boxplot graph in Fig. 3 for PRePAD-MMSE and PReP-AD-HVL methods

\begin{tabular}{cccccc}
\hline \multicolumn{5}{c}{ Error Rate Statistics } \\
\hline Month & No of Patients & Mean & Median & IQR & No of Outliers \\
\hline \multicolumn{5}{c}{ PReP-AD-MMSE } \\
\hline 12 & 115 & 3.47 & 2.59 & 3.05 & 6 \\
24 & 98 & 4.32 & 2.97 & 4.89 & 7 \\
36 & 88 & 4.25 & 3.17 & 4.32 & 3 \\
48 & 69 & 5.49 & 4.53 & 5.37 & 3 \\
60 & 33 & 6.25 & 5.05 & 5.11 & 2 \\
72 & 23 & 6.30 & 5.31 & 6.79 & 1 \\
\hline \multicolumn{7}{c}{ PReP-AD-HVL } \\
\hline 6 & 175 & 0.95 & 0.46 & 0.74 & 21 \\
12 & 175 & 1.63 & 0.91 & 1.48 & 21 \\
\hline
\end{tabular}

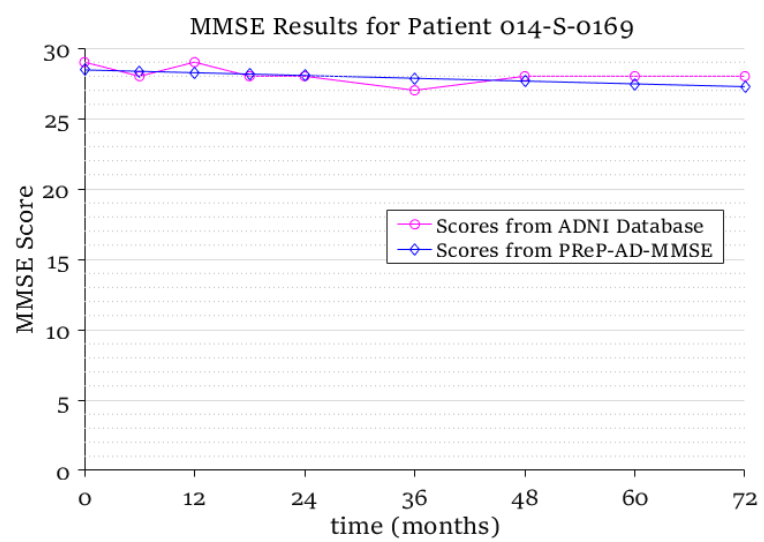

(a)

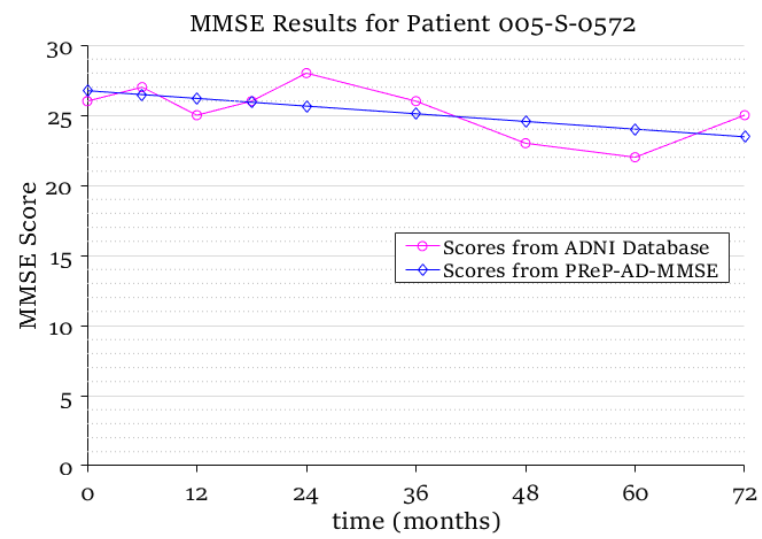

(b)

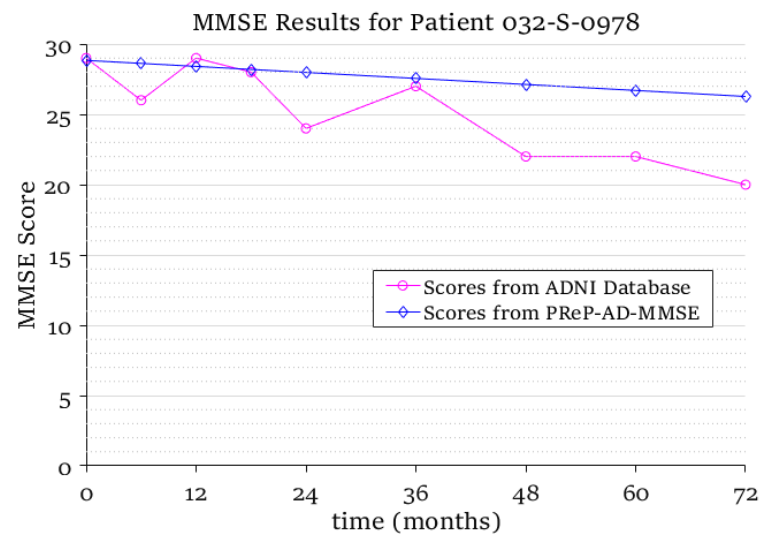

(c)

Figure 4: Examples of disease progression computed by PReP-ADMMSE for MCI patients with respect to the MMSE scores reported in ADNI database over a 72-month period with different average LOOCV error rates: (a) $1.9 \%$ for patient $014-\mathrm{S}-0169$, (b) $5.4 \%$ for patient $005-\mathrm{S}-0572$, and (c) $12.2 \%$ for patient $032-\mathrm{S}-0978$

In Fig. 4, we plot disease progression results computed by PRePAD-MMSE for three representative patients, chosen based on their different error rates. The MMSE scores reported in ADNI database are shown in pink, and the results computed by our PReP-AD-MMSE are marked in blue. In Fig. $4 \mathrm{a}$, an average error rate of $1.9 \%$ is observed for patient $014-\mathrm{S}-0169$ whose disease progression is considered stable. In Fig. 4b, we obtain a slightly larger average error 
rate of $5.4 \%$ for patient $005-\mathrm{S}-0572$, who shows a declining cognitive ability in time. The largest error rate among the three chosen patients is patient $032-\mathrm{S}-0978$ with an average error rate of $12.2 \%$ as shown in Fig. 4c. For patient 032-S-0978, the decrease rate in MMSE scores is larger than the rate computed by PReP-AD-MMSE.

\subsection{PReP-AD-HVL Results}

For PReP-AD-HVL method, disease progression is computed for 175 MCI patients. Although gene expressions were available for all patients in ADNI database, only 26 MCI patients have hippocampal volume measurements. With Algorithm 2, we estimated disease progression for the patients without the volume measurements using genetic similarity between patients. With the exception of three patients, PReP-AD-HVL computes disease progression as either stable or decreasing for 172 patients. We consider the disease progression for a patient to be stable when the total hippocampal volume change is less than $30 \mathrm{~mm}^{3}$ for a 12 -month period. PReP-AD-HVL computed stable progression for 29 patients. Disease progression is considered to be relatively significant when the total hippocampal volume loss is larger than $80 \mathrm{~mm}^{3}$ for 12 months, which was the case for 45 patients. For the remaining 98 patients, a moderate rate of decrease was computed by PReP-AD-HVL.

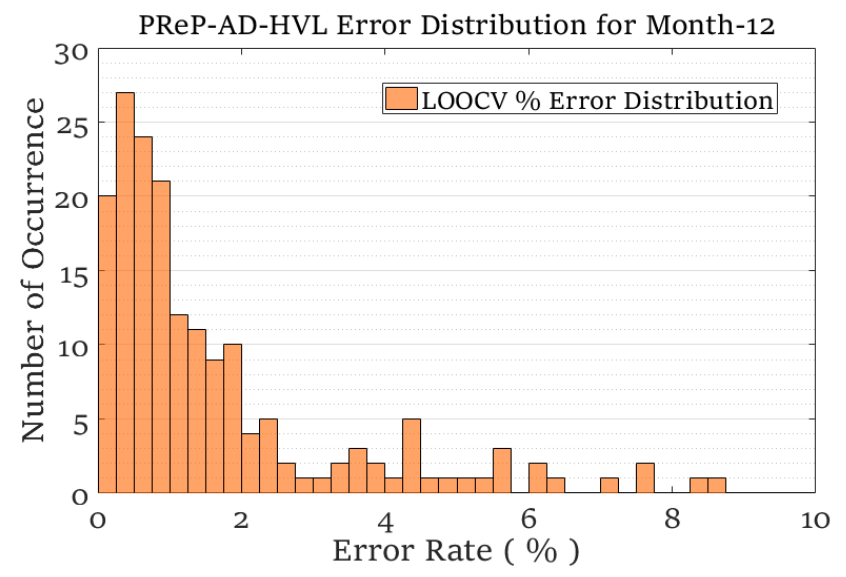

Figure 5: Histogram of LOOCV percentage error rates of PReP-ADHVL method for 175 MCI patients

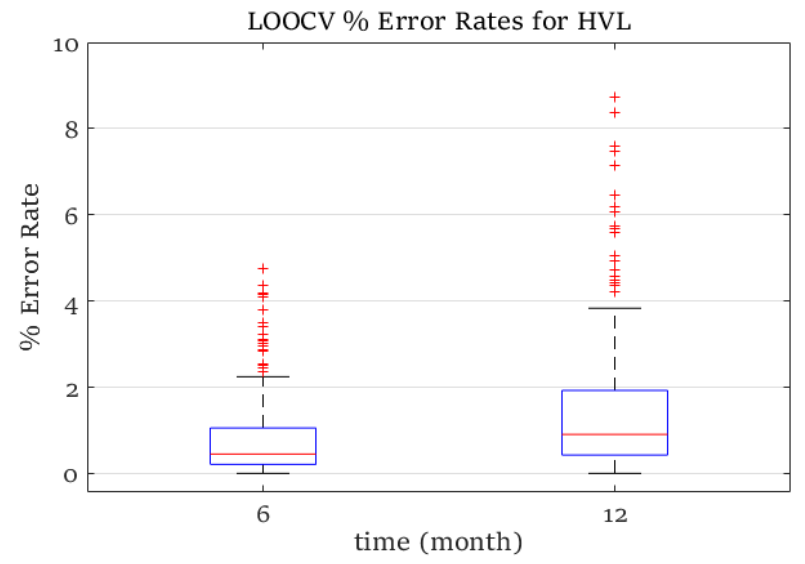

Figure 6: Boxplot graph of percentage LOOCV error rates of PRePAD-HVL method for a 12-month period
In Fig. 5, the histogram of percentage LOOCV error rates is illustrated for month 12 , where an average error rate of $1.63 \%$ is observed with a standard deviation of $1.81 \%$ for 175 patients. The distribution of error rates of PReP-AD-HVL for months 6 and 12 are shown with the boxplot graph in Fig. 6. Error rate statistics obtained from boxplot graph are listed in Table 2. The IQR, which is presented as a box in Fig. 6, is 0.74 and 1.48 for months 6 and 12, respectively.

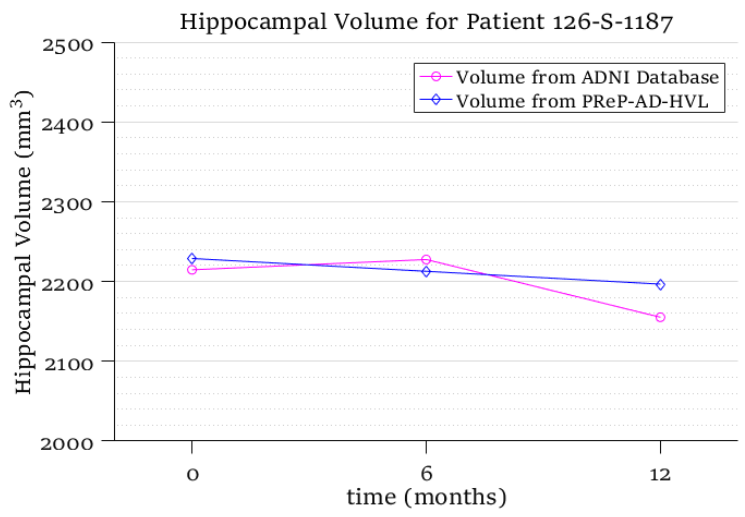

(a)

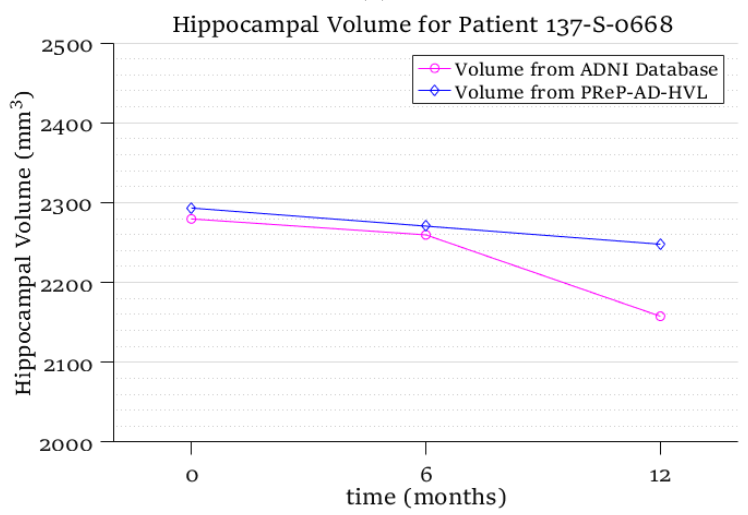

(b)

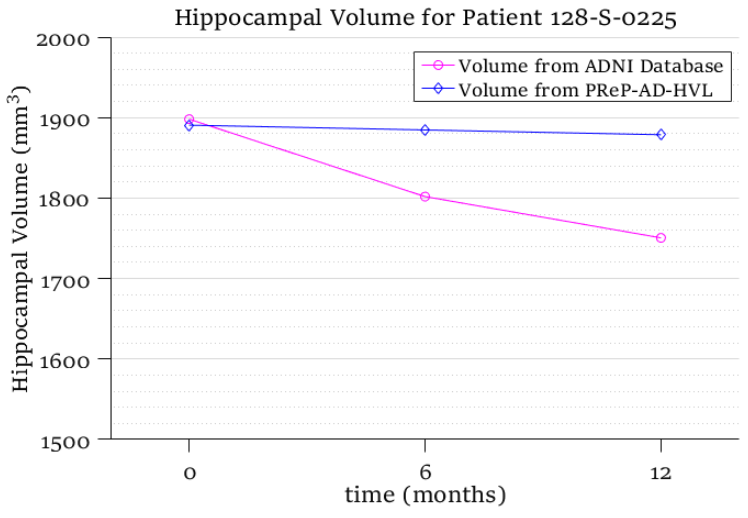

(c)

Figure 7: Examples of disease progression computed by PReP-ADHVL for MCI patients with respect to hippocampal volume measurements (average of left and right lobes) reported in ADNI database over a 12-month period with different error rates: (a) $1.24 \%$ for patient 126-S-1187, (b) $3.37 \%$ for patient 137-S-0668, and (c) $7.14 \%$ for patient $128-\mathrm{S}-0225$ 
For PReP-AD-HVL method, examples of disease progression results are shown in Fig. 7, where the hippocampal volume measurements from the database (average volume of left and right lobes) are in pink and the computed results are in blue. We calculate an error rate of $1.24 \%$ for patient $126-\mathrm{S}-1187$ as shown in Fig. 7a. In Fig. 7b, LOOCV error rate is obtained as $3.37 \%$ for patient $137-$ S-0668. A larger error rate of $7.14 \%$ is shown with Fig. 7c for patient 128-S-0225.

\subsection{Discussion}

One can visualize the distribution of LOOCV error rates for PREPAD methods using histograms (in Figs. 2 and 5) and boxplot graphs (in Figs. 3 and 6). From histograms, we analyze the frequency of error rates and the characteristics of error distributions, such as skewness, which is a measure of asymmetry. For PReP-AD-MMSE method, the error rate distribution is shown in Fig. 2a for month 12 and in Fig. $2 b$ for month 48 . The uniformity of the distribution of error rates for month 48 compared to month 12 implies that the amount of increase in the error rates obtained from PReP-AD-MMSE vary as time progresses. In other words, for some patients (approximately half of them), the small error rates obtained at month 12 remain the same at month 48 , whereas it increases in the range of $2 \%$ for remaining patients. In Fig. 5, regarding to the small mean value and the positive skewness in the distribution of error rates, we can conclude that for most of the MCI patients we obtain smaller error rates with PReP-AD-HVL method. The shorter time period during which hippocampal volume measurements are collected may be one of the reasons for this smaller error rate.

In a boxplot graph [41], a smaller IQR (i.e., a measure of spread) implies that the middle $50 \%$ of the dataset is closer to each other [42]. In Fig. 3, the distributions of LOOCV error rates computed for MMSE scores from MCI patients are presented with boxplots for distinct months. Even though an increasing trend is observed from month 12 to 72 in the value of IQR, a larger value is observed for month 24 with respect to the next time point (i.e., month 36 ). It can be concluded from Fig. 3 that for a larger percentage of patients PReP-AD-MMSE computes higher error rates at month 24. In Fig. 6, we generate boxplots for the distribution of error rates computed by PReP-AD-HVL. Even though the error distribution spread in a larger range at month 12 , the number of patients with extreme error rates does not change in time, which implies that the results computed by PReP-AD-MMSE are consistent over time.

\section{CONCLUDING REMARKS}

In this paper we present PReP-AD methods which are built based on artificial intelligence computation techniques to investigate the impact of gene expressions on AD progression. In addition to expressions of the genes that affect AD-related protein biomarkers, PReP-AD methods make use of the change in MMSE scores and the rate of hippocampal volume loss over time as metrics for cognitive changes in MCI patients. Disease progression computed by PRePAD-MMSE and PReP-AD-HVL methods based on MMSE scores and hippocampal volume loss are evaluated using our LOOCV based validation algorithm. We obtain an average error rate of $4.8 \%$ for PReP-AD-MMSE over a period of 72 months and $1.63 \%$ for PRePAD-HVL over 12 months.

The consistency of the results is encouraging to employ artificial intelligence based methods to build decision support tools for the computation of $\mathrm{AD}$ progression. We are planning to extend our study to include the AD patients who are administered AD-related drugs to evaluate drug efficacy on disease progression.

\section{ACKNOWLEDGEMENTS}

Data used in the preparation of this article were obtained from the Alzheimer's Disease Neuroimaging Initiative (ADNI) database (adni.loni.usc.edu) [13], launched in 2003 as a public-private partnership to test whether serial MRI, positron emission tomography (PET), other biological markers, and clinical and neuropsychological assessment can be combined to measure the progression of MCI and early AD. For up-to-date information, see www.adni-info.org.

Some of the initial results were collected by Carl Negro who is a graduate student at the City College of New York.

\section{REFERENCES}

[1] P Robert, S Ferris, S Gauthier, R Ihl, B Winblad, and F Tennigkeit. Review of alzheimers disease scales: is there a need for a new multi-domain scale for therapy evaluation in medical practice. Alzheimers Res Ther, 2(4):24, 2010.

[2] E Gallego-Jutglà, J Solé-Casals, F Vialatte, M Elgendi, A Cichocki, and J Dauwels. A hybrid feature selection approach for the early diagnosis of alzheimers disease. Journal of neural engineering, 12(1):016018, 2015.

[3] C R Jack, R C Petersen, Y Xu, P C Obrien, G E Smith, R J Ivnik, B F Boeve, E G Tangalos, and E Kokmen. Rates of hippocampal atrophy correlate with change in clinical status in aging and ad. Neurology, 55(4):484-490, 2000.

[4] C Laske, H R Sohrabi, S M Frost, K López-de Ipiña, P Garrard, M Buscema, J Dauwels, S R Soekadar, S Mueller, C Linnemann, et al. Innovative diagnostic tools for early detection of alzheimer's disease. Alzheimer's \& Dementia, 11(5):561-578, 2015.

[5] P M Thompson, K M Hayashi, G I De Zubicaray, A L Janke, S E Rose, J Semple, M S Hong, D H Herman, D Gravano, D M Doddrell, and A W Toga. Mapping hippocampal and ventricular change in Alzheimer's Disease. Neuroimage, 22(4), 2004.

[6] R E Kennedy, G R Cutter, G Wang, and L S Schneider. Using baseline cognitive severity for enriching alzheimer's disease clinical trials: How does mini-mental state examination predict rate of change? Alzheimer's \& Dementia: Translational Research \& Clinical Interventions, 1(1):46-52, 2015.

[7] Y Stern, X Liu, M Albert, J Brandt, D M Jacobs, C Del Castillo-Castaneda, K Marder, K Bell, M Sano, F Bylsma, et al. Application of a growth curve approach to modeling the progression of alzheimer's disease. The Journals of Gerontology Series A: Biological Sciences and Medical Sciences, 51(4):M179-M184, 1996.

[8] C Laske, T Leyhe, E Stransky, N Hoffmann, A J Fallgatter, and J Dietzsch. Identification of a blood-based biomarker panel for classification of alzheimer's disease. International Journal of Neuropsychopharmacology, 14(9):1147-1155, 2011.

[9] K Blennow. Cerebrospinal fluid protein biomarkers for alzheimer's disease. NeuroRx, 1(2):213-225, 2004.

[10] P R Walker, B Smith, Q Y Liu, A F Famili, J J Valdés, Z Liu, and $B$ Lach. Data mining of gene expression changes in alzheimer brain. Artificial intelligence in medicine, 31(2):137-154, 2004.

[11] N Schuff, N Woerner, L Boreta, T Kornfield, L M Shaw, J Q Trojanowski, P M Thompson, C R Jack, M W Weiner, Disease Neuroimaging Initiative, et al. Mri of hippocampal volume loss in early alzheimer's disease in relation to apoe genotype and biomarkers. Brain, 132(4):1067-1077, 2009.

[12] T-T Huang, Y Zou, and R Corniola. Oxidative stress and adult neurogenesis effects of radiation and superoxide dismutase deficiency. In Seminars in cell \& developmental biology, volume 23, pages 738-744. Elsevier, 2012.

[13] ADNI - Alzheimer's Disease Neuroimaging Initiative. National Institutes of Health, (for up-to-date information, see https://www.adni-info.org).

[14] J Kusyk, C S Sahin, M U Uyar, E Urrea, and S Gundry. Self organization of nodes in mobile ad hoc networks using evolutionary games and genetic algorithms. J. of Advanced Research, 2(3):253264, 2011.

[15] C S Sahin, M U Uyar, S Gundry, and E Urrea. Self organization for area coverage maximization and energy conservation in mobile ad hoc networks. In Transactions on Computational Science $X V$, pages 
49-73. Springer, 2012.

[16] A Saribudak, Y Dong, J Hsieh, and M U Uyar. Bio-inspired computation approach for tumor growth with spatial randomness analysis of kidney cancer xenograft pathology slides. In 9th EAI Conference on Bio-inspired Information and Communications Technologies (BICT 2015), pages 1-8. IEEE, 2015.

[17] L Wang, J S Swank, I E Glick, M H Gado, M I Miller, J C Morris, and J G Csernansky. Changes in hippocampal volume and shape across time distinguish dementia of the alzheimer type from healthy aging? Neuroimage, 20(2):667-682, 2003.

[18] M F Folstein, S E Folstein, and P R McHugh. Mini-mental state: a practical method for grading the cognitive state of patients for the clinician. Journal of psychiatric research, 12(3):189-198, 1975.

[19] P A Wilkosz, H J Seltman, B Devlin, E A Weamer, O L Lopez, S T DeKosky, and R A Sweet. Trajectories of cognitive decline in alzheimer's disease. International Psychogeriatrics, 22(02):281-290, 2010.

[20] M S Mendiondo, JW Ashford, R J Kryscio, and F A Schmitt. Modelling mini mental state examination changes in alzheimer's disease. Statistics in medicine, 19(11-12):1607-1616, 2000.

[21] B D Hoyt, P J Massman, C Schatschneider, N Cooke, and R S Doody. Individual growth curve analysis of apoe $\varepsilon 4$-associated cognitive decline in alzheimer disease. Archives of neurology, 62(3):454-459, 2005.

[22] J O Brooks, H C Kraemer, E D Tanke, and J A Yesavage. The methodology of studying decline in alzheimer's disease. Journal of the American Geriatrics Society, 41(6):623-628, 1993.

[23] A Saribudak, E Ganic, J Zou, S Gundry, and M U Uyar. Toward genomic based personalized mathematical models for breast cancer tumor growth. In IEEE 14th Int'l. Conf. on BioInformatics and BioEngineering (BIBE), pages 115-119, Nov. 2014.

[24] A Saribudak, S Gundry, J Zou, and M U Uyar. Genomic based personalized chemotherapy analysis to support decision systems for breast cancer. In Medical Measurements and Applications (MeMeA), 2015 IEEE Intl. Symp. on, pages 495-500. IEEE, 2015.

[25] A Saribudak, S Gundry, J Zou, and M U Uyar. A gene expression-based mathematical modeling approach for breast cancer tumor growth and shrinkage. Network Modeling Analysis in Health Informatics and Bioinformatics, 4(1):1-13, 2015.

[26] A Saribudak, Y Dong, S Gundry, J Hsieh, and M U Uyar. Mathematical models of tumor growth using voronoi tessellations in pathology slides of kidney cancer. In Engineering in Medicine and Biology Society (EMBC), 2015 37th Annual International Conference of the IEEE, pages 4454-4457. IEEE, 2015.

[27] A Saribudak, H Veterduy, K Hubbard, and M U Uyar. Heterogeneity of colorectal cancer cell positions as a cell viability biometric. In Proc. IEEE EMBS Int'l. Conference on Biomedical and Health
Informatics (BHI), pages 21-24, Feb. 2016.

[28] A Saribudak, H Veterduy, K Hubbard, and M U Uyar. Quantification of cell apoptosis for in-vitro colorectal cancer cell cultures based on morphological features. In Proc. IEEE EMBS Int'l. Conference on Biomedical and Health Informatics (BHI), pages 82-85, Feb. 2016.

[29] S Norton, F E Matthews, D E Barnes, K Yaffe, and C Brayne. Potential for primary prevention of alzheimer's disease: an analysis of population-based data. The Lancet Neurology, 13(8):788-794, 2014.

[30] S G Mueller, M W Weiner, L J Thal, R C Petersen, C R Jack, W Jagust, J Q Trojanowski, A W Toga, and L Beckett. Ways toward an early diagnosis in alzheimer's disease: The alzheimer's disease neuroimaging initiative (adni). Alzheimers Dementia, 1(1), 2005.

[31] Alzheimer's Association et al. 2013 alzheimer's disease facts and figures. Alzheimer's \& dementia, 9(2):208-245, 2013.

[32] T N Tombaugh and N J McIntyre. The mini-mental state examination: a comprehensive review. Journal of the American Geriatrics Society, 40(9):922-935, 1992.

[33] E Franko, O Joly, and for the Alzheimer's Disease Neuroimaging Initiative (2013). Evaluating alzheimer's disease progression using rate of regional hippocampal atrophy. PLoS ONE, 8(8), 2013.

[34] W J Henneman, J D Sluimer, J Barnes, W M van der Flier, I C Sluimer, N C Fox, P Scheltens, H Vrenken, and F Barkhof. Hippocampal atrophy rates in alzheimer disease: added value over whole brain volume measures. Neurology, 72(11), 2009.

[35] R Edgar, M Domrachev, and A E Lash. Gene expression omnibus: Ncbi gene expression and hybridization array data repository. Nucleic acids research, 30(1):207-210, 2002.

[36] NBIA - National Biomedical Imaging Archive. National Institutes of Health, https://imaging.nci.nih.gov/ncia.

[37] S Gundry, J Zou, E Urrea, C S Sahin, J Kusyk, and M U Uyar. Analysis of emergent behavior for ga-based topology control mechanism for self-spreading nodes in MANETs. In Advances in Intelligent Modelling and Simulation, volume 422 of Studies in Computational Intelligence, pages 155-183. Springer Berlin Heidelberg, 2012.

[38] K Price, R M Storn, and J A Lampinen. Differential evolution: a practical approach to global optimization. Springer Science \& Business Media, 2006.

[39] J Demšar. Statistical comparisons of classifiers over multiple data sets. The Journal of Machine Learning Research, 7:1-30, 2006.

[40] S Arlot, A Celisse, et al. A survey of cross-validation procedures for model selection. Statistics surveys, 4:40-79, 2010.

[41] J L Hintze and R D Nelson. Violin plots: a box plot-density trace synergism. The American Statistician, 52(2):181-184, 1998.

[42] M Krzywinski and N Altman. Points of significance: visualizing samples with box plots. Nature methods, 11(2):119-120, 2014. 